\title{
Development of ethosomes with taguchi robust design-based studies for transdermal delivery of alfuzosin hydrochloride
}

\author{
D. Prasanthi, *P. K. Lakshmi \\ G. Pulla Reddy College of Pharmacy, Osmania University, Hyderabad, India
}

\begin{abstract}
In the present investigation efficiency of ethosomes as novel lipid carriers for transdermal delivery of Alfuzosin Hydrochloride (AH) has been evaluated. Taguchi robust design method was used for optimization of ethosomal formulations. Phospholipid type, concentration of phospholipid and concentration of ethanol was selected as independent variables and their effect on the dependent variables (entrapment efficiency and flux) was studied. Ethosomal formulation (EA8) with soya phosphatidylcholine (3\%) and ethanol $20 \%$ were optimized. Vesicles were spherical, unilamellar with smooth surface. The optimized formulation exhibited vesicle size $(6.85 \pm 1.35 \mu \mathrm{m})$, zeta potential $(-8.14 \pm 0.62 \mathrm{mv})$, entrapment efficiency $(91.86 \pm 3.25 \%)$, flux $\left(27.42 \pm 0.04 \mu \mathrm{g} / \mathrm{cm}^{2} / \mathrm{hr}\right)$, lag time $(0.26 \pm 0.20 \mathrm{hr})$ and skin deposition $(298.01 \pm 15.4 \mu \mathrm{g} / \mathrm{g})$. Transdermal flux was enhanced by 6.92 times over drug solution. Vesicle skin interaction studies showed fatty change in the dermis. The formulations were stable at $4^{\circ} \mathrm{C}$ for 120 days. Results suggested ethosomes as efficient carriers for AH transdermal delivery.
\end{abstract}

Key Words: DOE, vesicles, ethanolic vesicles, permeation enhancement, BPH.

\section{INTRODUCTION}

Transdermal drug delivery (TDD) is the potential route for delivering systemic drugs. But the greatest challenge is the barrier nature of stratum corneum (Aqil et al., 2007). Many techniques have been aimed to disrupt or weaken the barrier property of skin. One method is the use of vesicle formulations as skin delivery systems (Honeywell-Nguyen and Bouwstra, 2005). Intensive research over the past two decades led to the development of novel carriers, the ethanolic liposomes that have been termed ethosomes (Touitou et al., 2000).

Ethosomes are novel lipid vesicles embodying high concentration (20-45\%) of ethanol and are prepared by dissolving the lipids and drug in ethanol (Dayan and Touitou, 2000). Several studies have reported that ethosomes were able to improve in vitro and in vivo skin delivery of various drugs, both under

\footnotetext{
*Corresponding Author:

Dr. P. K. Lakshmi, M. Pharm, PhD

Professor \& Head, Department of Pharmaceutics

G. Pulla Reddy College of Pharmacy

Osmania University, Hyderabad, India- 500028

E-mail: drlakshmisuresh@gmail.com

Contact No.: 09000044452
}

occlusive and non-occlusive conditions (Prasanthi and Lakshmi, 2012).

The aim of the present study was to statistically optimize the vesicular formulations (Ethosomes) for enhanced skin delivery of a model drug Alfuzosin hydrochloride $(\mathrm{AH}), \alpha$-adrenoreceptor antagonist used for benign prostatic hyperplasia. It has low oral bioavailability of about $64 \%$ and its physicochemical properties like molecular weight (425.9), half-life (3-5), $\log P$ value (1.604), and low dose (2.5 to $10 \mathrm{mg} /$ day) makes it an ideal drug candidate for TDD (Martindale, 1993). AH has been previously identified as a promising candidate for TDD (Prasanthi et al., 2010).

Taguchi robust design is a statistical technique which studies all levels of input parameters with fewer experiments and optimizes the experiment having least variability. The variability of a property is expressed by signal to noise ratio (S/N Ratio) (Kim et al., 2007).

In the present study, ethosomes bearing $\mathrm{AH}$ was prepared by cold method and optimized by Taguchi 
robust design method. They were evaluated for permeation enhancement over pure drug solution and the optimized formulation was evaluated for vesicle skin interaction studies and stability studies.

\section{MATERIAL AND METHOD}

Alfuzosin hydrochloride (AH) was obtained as a gift sample from Dr. Reddy's Laboratories Ltd (Hyderabad, India). Phospholipon $90 \mathrm{H}$ and phospholipon 80 $\mathrm{H}$ was obtained as a gift sample from Lipoid $\mathrm{GmbH}$ (Germany). Soyaphosphatidyl choline (SPC) was purchased from Otto Chem. Ltd. (India). Ethanol was purchased from S. D. Fine-Chem. Ltd. (India).

\section{Preparation of ethosomes}

Ethosomal formulations were prepared by the cold method (Verma and Pathak, 2012) using Taguchi robust design. The ethanolic vascular system was composed of phospholipid (2.0\% to $4.0 \%$ with/Vol), ethanol $(20 \%$ to $40 \% \mathrm{Vol} / \mathrm{Vol})$, drug (AH, $0.5 \%$ $\mathrm{wt} / \mathrm{Vol})$ and double distilled water to $100 \%$ (Vol/Vol). Phospholipid was dissolved along with the drug in ethanol. This mixture was heated to $40^{\circ} \mathrm{C} \pm 1^{\circ} \mathrm{C}$ and a fine stream of double-distilled water was added slowly, with constant mixing at $700 \mathrm{rpm}$ with a mechanical stirrer (Remi Instruments, Vasai, India) in a house-built closed container. Mixing was continued for an additional 5 minutes, while maintaining the system at $40^{\circ} \mathrm{C} \pm$ $1^{\circ} \mathrm{C}$. The preparation was left to cool at room temperature for $30 \mathrm{~min}$ and then it was sonicated at $4^{\circ} \mathrm{C}$ for five cycles of 3 minutes each with a 1-minute rest between cycles using a probe sonicator (SonicsVibra Cell). Nine formulations were prepared
Table 1: Three-factor, three-level Taguchi L9 Orthogonal array experimental design.

\begin{tabular}{lccc}
\hline \multirow{2}{*}{ Factors } & \multicolumn{3}{c}{ Levels } \\
\cline { 2 - 4 } & $\mathbf{1}$ & $\mathbf{2}$ & $\mathbf{3}$ \\
\hline $\begin{array}{l}\text { A. Type of phospholi- } \\
\text { pid }\end{array}$ & $80 \mathrm{H}$ & $90 \mathrm{H}$ & $\begin{array}{c}\text { soya phosphatidyl } \\
\text { choline }\end{array}$ \\
$\begin{array}{l}\text { B. Concentration of } \\
\text { phospholipid (\%) }\end{array}$ & 2 & 3 & 4 \\
$\begin{array}{l}\text { C. Concentration of } \\
\text { ethanol (\%) }\end{array}$ & 20 & 30 & 40 \\
\hline $\begin{array}{l}\text { Response : Entrapment efficiency and Flux }\left(\mathrm{Jss}_{\mathrm{ss}} \mu \mathrm{g} / \mathrm{cm}^{2} / \mathrm{hr}\right) . \\
\end{array}$
\end{tabular}

according to Taguchi L9 orthogonal array design shown in Table 2. Ethosomes were compared with thirty percent of hydroethanolic solution of drug and drug solution in distilled water.

\section{Experimental design}

A three-factor, three-level Taguchi L9 orthogonal array experimental design was constructed using MINITAB 16 software (Minitab Inc., PA, U.S.A). The independent variables selected were the type of phospholipid, concentration of phospholipid and concentration of ethanol. Their corresponding levels and the dependent variables are shown in Table1.

\section{Evaluation of ethosomes}

Vesicular shape and surface morphology

Scanning electron microscopy (SEM) was used to characterize the surface morphology of the prepared vesicles. One drop of ethosomal suspension was mounted on a clear-glass stub, air-dried, gold coated with Polaron E5100 sputter coater (Polaron, United Kingdom), and visualized under scanning electron microscope (Jeol 5400, Japan).

Table 2: Observed responses in Taguchi Robust Design for AH ethosomal formulation (EA).

\begin{tabular}{cccccc}
\hline \multirow{2}{*}{$\begin{array}{c}\text { Formulation } \\
\text { code }\end{array}$} & \multicolumn{3}{c}{ Independent variables } & \multicolumn{2}{c}{ Dependent variables } \\
\cline { 2 - 5 } & $\mathbf{A}$ & $\mathbf{B ~ ( \% )}$ & $\mathbf{C ~ ( \% )}$ & $\mathbf{Y}_{\mathbf{1}} \mathbf{( \% )}$ & $\mathbf{Y}_{\mathbf{2}}\left(\mathbf{\mu g} / \mathbf{c m}^{2} / \mathbf{h r}\right)$ \\
\hline EA1 & 1 & 1 & 1 & $65.73 \pm 6.14$ & $18.41 \pm 0.41$ \\
EA2 & 1 & 2 & 2 & $82.86 \pm 4.20$ & $26.71 \pm 0.15$ \\
EA3 & 1 & 3 & 3 & $74.5 \pm 3.37$ & $24.03 \pm 0.21$ \\
EA4 & 2 & 1 & 2 & $60.03 \pm 5.75$ & $15.98 \pm 0.24$ \\
EA5 & 2 & 2 & 3 & $79.1 \pm 3.57$ & $24.54 \pm 0.19$ \\
EA6 & 2 & 3 & 1 & $63.96 \pm 4.56$ & $21.60 \pm 0.16$ \\
EA7 & 3 & 1 & 3 & $84.50 \pm 4.05$ & $25.93 \pm 0.16$ \\
EA8 & 3 & 2 & 1 & $91.86 \pm 3.25$ & $27.42 \pm 0.04$ \\
EA9 & 3 & 3 & 2 & $76.00 \pm 2.28$ & $23.18 \pm 0.41$ \\
\hline
\end{tabular}

A: Type of phospholipid; B: Concentration of phospholipid (\%); C: Concentration of ethanol (\%);

$\mathrm{Y}_{1}$ :Entrapment efficiency (\%); $\mathrm{Y}_{2}:$ Flux $\left(\mu \mathrm{g} / \mathrm{cm}^{2} / \mathrm{hr}\right)$. Values represent mean $\pm \mathrm{SD}(\mathrm{n}=3)$. 
Table 3: Characterization of various formulations of $\mathrm{AH}$.

\begin{tabular}{lccc}
\hline Parameters & Ethosomes (3\% SPC, 20\% EtOH) & Hydroethanolic solution & Drug solution \\
\hline Shape and surface morphology & Spherical, unilamellar & - & - \\
Particle size $(\mu \mathrm{m})^{*}$ & $6.85 \pm 1.35$ & - & - \\
Zeta potential $(\mathrm{mV})^{*}$ & $-8.14 \pm 0.62$ & - & - \\
Entrapment efficiency $(\%)^{*}$ & $91.86 \pm 3.25$ & - & - \\
Transdermal flux $\left(\mu \mathrm{g} / \mathrm{cm}^{2} / \mathrm{hr}\right)^{*}$ & $27.42 \pm 0.04$ & $6.66 \pm 0.17$ & $3.96 \pm 0.13$ \\
Permeability co-efficient & $5.48 \pm 0.009$ & $0.13 \pm 0.03$ & $0.079 \pm 0.02$ \\
$(\mathrm{~cm} / \mathrm{hr}) \times 10^{-3} *$ & $0.26 \pm 0.20$ & $3.03 \pm 0.5$ & $4.06 \pm 0.25$ \\
Lag time $(\mathrm{hr})^{*}$ & 6.92 & 1.68 & - \\
Enhancement ratio & $298.01 \pm 15.4$ & $120.36 \pm 8.0$ & $86.33 \pm 11.99$ \\
Skin deposition $(\mu \mathrm{g} / \mathrm{g})^{*}$ & &
\end{tabular}

*-Values represent mean \pm SD $(n=3)$. EtOH: ethanol; SPC: soya phosphatidylcholine.

\section{Vesicle size and zeta potential}

The vesicle size and zeta potential were measured by photon correlation spectroscopy (Delsa Nano, Beckman Coulter Inc. UK).

\section{Entrapment efficiency}

Prepared ethosomal vesicles were separated from the free (unentrapped) drug by ultracentrifugation technique (Verma and Pathak, 2012). $2 \mathrm{ml}$ of the ethosomal suspension was diluted with distilled water up to $5 \mathrm{ml}$ and centrifuged at 20,000 rpm for $45 \mathrm{~min}$ at $4^{\circ} \mathrm{C}$ using a cooling centrifuge (Eltek centrifuge). After centrifugation, the supernatant and sediment were recovered, and sediment was lysed using methanol and filtered through a $0.45 \mu \mathrm{m}$ nylon disk filter. The concentration of $\mathrm{AH}$ in the supernatant and sediment was analysed by UV-VIS double beam spectrophotometer (Chemito Spectrascan UV2600, India) at $245 \mathrm{~nm}$. The percentage drug entrapment was calculated using the following equation:

$\%$ Drug entrapment $=\frac{\text { Amount of entrapped drug recovered }}{\text { Total amount of drug }} \times 100$

The determination of entrapment efficiency was repeated three times per sample at $25^{\circ} \mathrm{C}$.

\section{Skin permeation studies}

Institutional animal ethical committee (IAEC) approved skin permeation studies. Locally fabricated Keshary Chein diffusion cell was used for

Table 4: Stability of AH ethosomes.

\begin{tabular}{lcc}
\hline Parameters & Initially & After $\mathbf{1 2 0}$ days $\left(\mathbf{4} \pm \mathbf{1}^{\circ} \mathbf{C}\right)$ \\
\hline Vesicle size $(\mu \mathrm{m})$ & $6.85 \pm 1.35$ & $6.99 \pm 3.56$ \\
Zeta potential $(\mathrm{mV})$ & $-8.14 \pm 0.62$ & $-7.86 \pm 1.23$ \\
Entrapment efficiency $(\%)$ & $91.86 \pm 3.25$ & $90.98 \pm 2.87$ \\
\hline
\end{tabular}

permeation studies. Studies were conducted using dermatomed and prepared rat skin. $20 \mathrm{ml}$ of PBS 7.4 was taken in receptor compartment and was continuously stirred with a magnetic stirrer and equilibrated at $37^{\circ} \pm 1^{\circ} \mathrm{C}$ with a recirculating water bath. The dermatomed skin was mounted with stratum corneum facing upward into the donor compartment. $1 \mathrm{ml}$ of ethosomal formulation was taken in donor compartment and covered with parafilm to avoid any evaporation process. $1 \mathrm{ml}$ samples were withdrawn through the sampling port at predetermined intervals over $24 \mathrm{hr}$ and analysed for drug content by UV-VIS double beam spectrophotometer (Chemito Spectrascan UV2600, India) at $245 \mathrm{~nm}$. Similar studies were performed with hydroethanolic solution and drug solution.

\section{Skin deposition studies}

The amount of $\mathrm{AH}$ retained in the skin was deter-

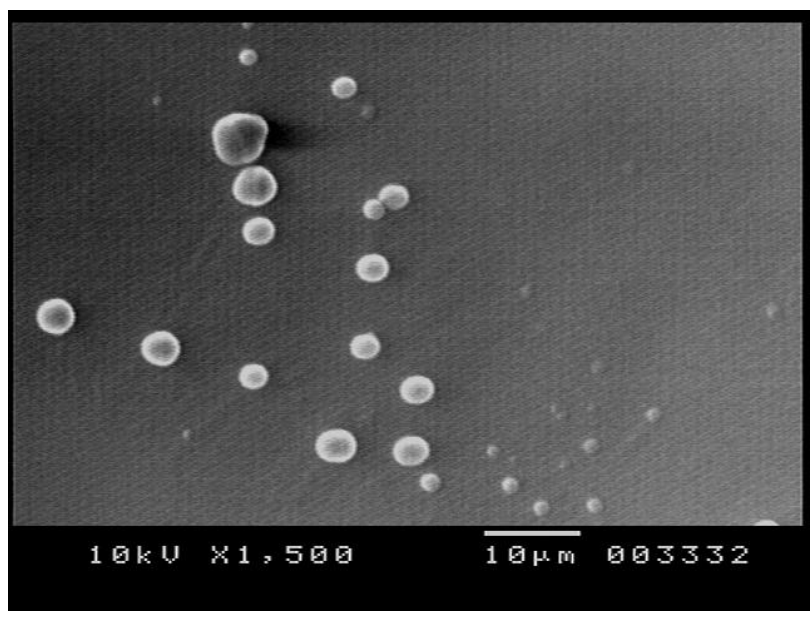

Figure 1: Scanning electron microscope photomicrograph of optimized ethosomal formulation EA8. 


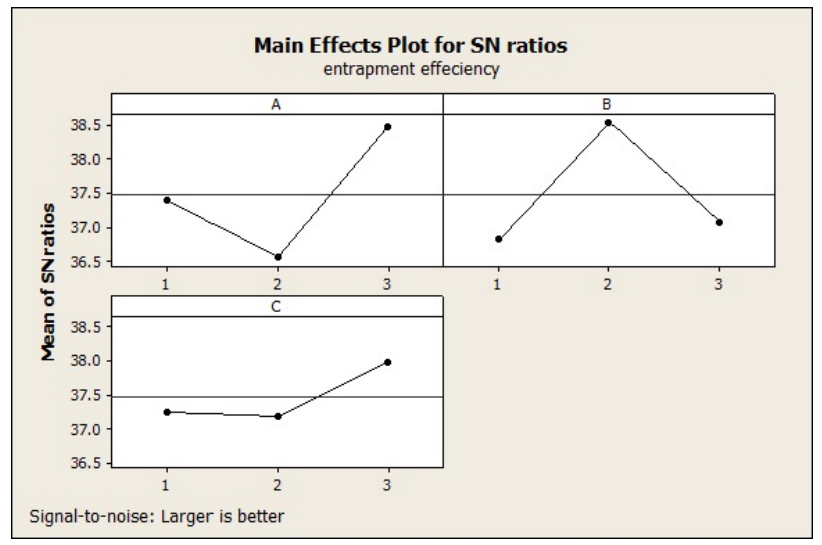

Figure 2: Main effects plot for $\mathrm{SN}$ ratios of $\mathrm{A}$ ) type of phospholipid, B) concentration of phospholipid and C) concentration of ethanol for entrapment efficiency.

mined by skin deposition studies. At the end of permeation studies $(24 \mathrm{hr})$, the skin was washed 10 times with a cloth immersed in methanol. A sample of skin was weighed and homogenized with methanol for $5 \mathrm{~min}$ using an electric stirrer. The resulting solution was centrifuged at $7000 \mathrm{rpm}$ for $10 \mathrm{~min}$ and supernatant was analysed for drug content by UV-VIS double beam spectrophotometer (Chemito Spectrascan UV2600, India) at 245nm.

\section{Vesicle-skin interaction studies}

Interaction studies were studied on the basis of structural changes in stratum corneum, epidermis and dermis (Jain et al., 2007). The formulations were applied topically to the dorsal portion of rats for $8 \mathrm{hrs}$. Later, the rats were killed by cervical dislocation; the skin was excised and fixed by immersion in $50 \%$ neutral formalin solution in saline for $24 \mathrm{hrs}$. The samples were subjected to histological processing, by dehydration and rehydration with graded alcohols, paraffin block processing and stained with haemotoxyllin-eosin. Microscopic evaluation using dark-light microscope was performed by a blinded assessor.

\section{Stability studies}

Vesicles were stored at $4^{\circ} \mathrm{C} \pm 0.5^{\circ} \mathrm{C}$ for 120 days. To determine their stability vesicle size, zeta potential and entrapment efficiency of the vesicles was measured using the method described earlier.

\section{Data analysis}

All the experiments were performed in triplicate unless specified, and the data are expressed as mean

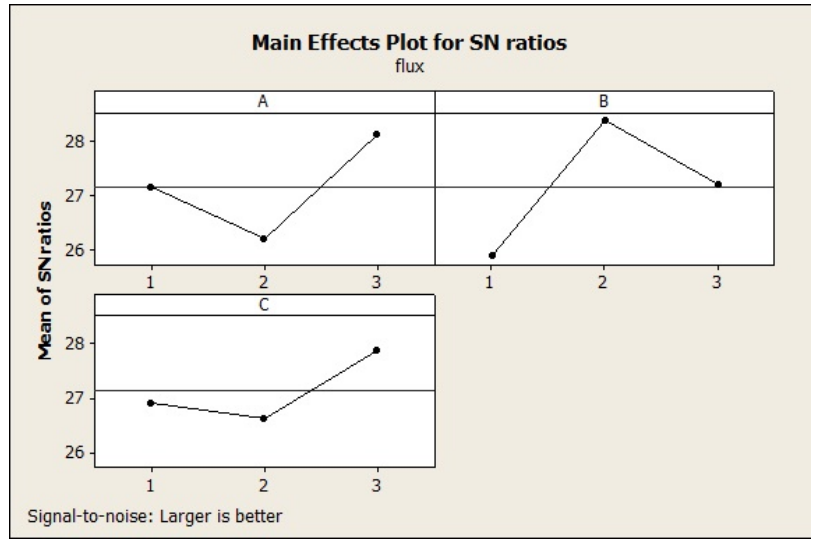

Figure 3: Main effects plot for $\mathrm{SN}$ ratios of $\mathrm{A}$ ) Type of phospholipid, B) concentration of phospholipid and C) concentration of ethanol for Flux.

values \pm standard deviations. Statistical significance was performed using one-way ANOVA at confidence limit of $P<0.05$. (MINITAB 16 software (Minitab Inc., PA, U.S.A)).

\section{RESULTS AND DISCUSSION}

Ethosomal formulations were prepared by the cold method according to Taguchi L9 orthogonal array design given in Table 2. On characterization spherical, unilamellar vesicles with smooth surface were observed under scanning electron microscope (Figure 1).

Ethosomes were optimized by varying phospholipid type, concentration of phospholipid and concentration of ethanol. Effect of independent variables on dependent variables was evaluated by SN ratio plots (Figure 2 \& Figure 3). Main effects plot of SN ratios showed phospholipid type had no significant effect on entrapment and flux (P>0.05). But maximum permeation was observed with soya phosphatidylcholine as it offers rigidity to the layers thereby reducing vesicle fusion resulting in decreased particle size and better permeation through skin pores.

Concentration of phospholipid ( $2 \%$ to $4 \%$ ) effected entrapment efficiency and flux. Previous studies reported increases in phospholipid, increased entrapment (Patel and Patel, 2009; Dubey et al., 2010). From the SN ratios plot it has been observed entrapment efficiency and flux increased with increase in phospholipid concentration up to $3 \%$, but further increase decreased entrapment efficiency 


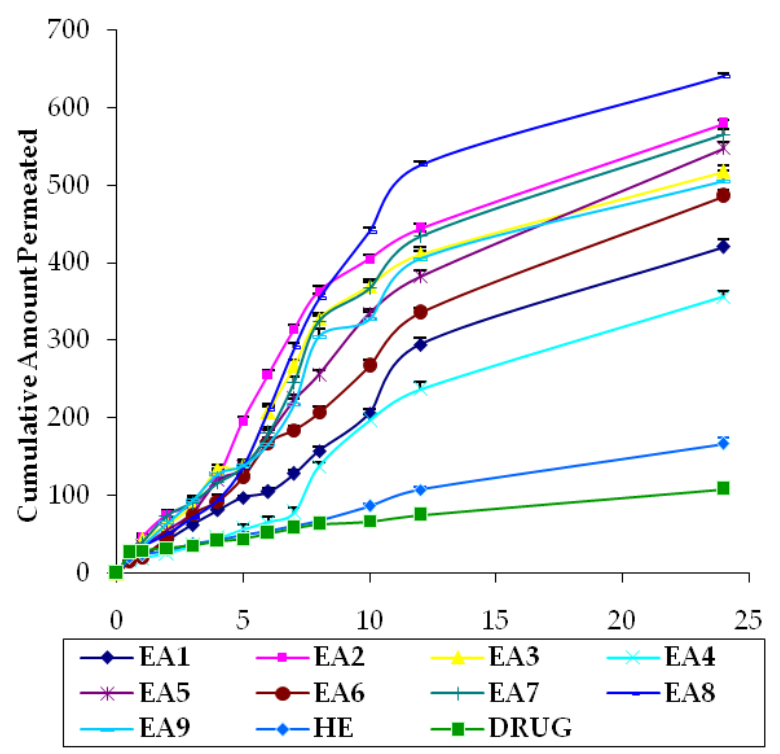

Figure 4: Cumulative amount permeated from ethosomal formulations, hydroethanolic solution (HE) and drug solution.

and flux. This effect is not significant ( $P>0.05)$. Concentration of ethanol (20\% to $40 \%$ ) showed linear relationship, as the concentration increased the entrapment and flux increased.

Permeation studies were performed using a rat abdominal skin. Overall ethosomes enhanced transdermal permeation by 4.03 to 6.92 times when compared with drug solution. Higher skin retention of the drug was seen with ethosomes $(298.01 \pm 15.4$ $\mu \mathrm{g} / \mathrm{g}$ to $1543.96 \pm 40.1 \mu \mathrm{g} / \mathrm{g}$ ). Ethosomal formulation EA8 were optimized, which showed maximum flux of $27.42 \pm 0.04 \mu \mathrm{g} / \mathrm{cm}^{2} / \mathrm{hr}$; lower lag time $0.26 \pm 0.20$ $\mathrm{hr}$ and higher entrapment efficiency of $91.86 \pm 3.25 \%$ when compared with other formulations. Cumulative amount permitted by ethosomal formulations ranged from $356.68 \pm 7.62 \mu \mathrm{g} / \mathrm{cm}^{2}$ to $637.10 \pm 2.95$ $\mu \mathrm{g} / \mathrm{cm}^{2}$ when compared with hydroethanolic solution $\left(167.13 \pm 6.92 \mu \mathrm{g} / \mathrm{cm}^{2}\right)$ and drug solution $\left(108.35 \pm 2.85 \mu \mathrm{g} / \mathrm{cm}^{2}\right)$. Increased flux (15.98 \pm 0.24 $\mu \mathrm{g} / \mathrm{cm}^{2} / \mathrm{hr}$ to $27.42 \pm 0.04 \mu \mathrm{g} / \mathrm{cm}^{2} / \mathrm{hr}$ ) and lower lag time $(0.26 \pm 0.20 \mathrm{hr})$ was observed with ethosomes when compared with drug solution $(3.96 \pm 0.13$ $\mu \mathrm{g} / \mathrm{cm}^{2} / \mathrm{hr} ; 4.06 \pm 0.25 \mathrm{hr}$ ) and hydroethanolic solution $\left(6.66 \pm 0.17 \mu \mathrm{g} / \mathrm{cm}^{2} / \mathrm{hr} ; 3.03 \pm 0.5 \mathrm{hr}\right)$. Comparison of cumulative amount permeated of various formulations is given in Figure 4. The results are given in Table 3.

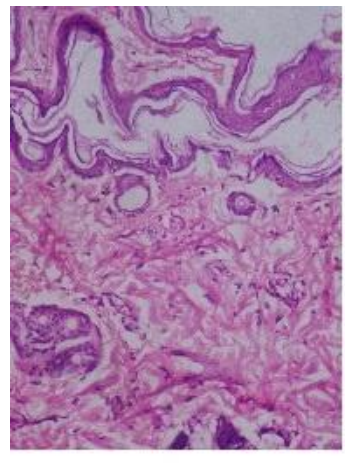

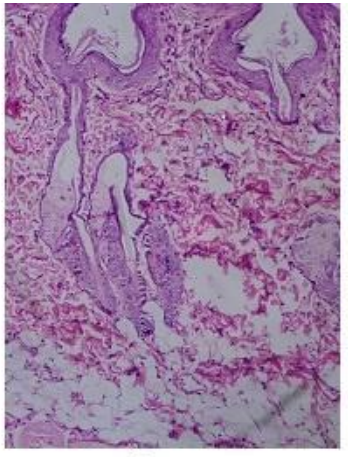

$\mathrm{b}$
Figure 5: Histological section of skin a) control and b) treated with ethosomal formulation (EA8) under magnification $\times 200$.

When compared to drug solution and hydroethanolic solution ethosomes showed better permeation due to the synergistic effect of phospholipids and ethanol as phospholipids in the vesicles entrap hydrophilic/lipophilic drug molecules act as carriers and deliver the entrapped drugs into or across the skin. Ethanol enhances permeation by various mechanisms such as push and pull effect, fluidization of stratum corneum lipids and vesicular lipid bilayers providing malleability to vesicles to penetrate skin pores smaller than their diameter (Panchagnula et al., 2001; Kowatsu and Okada, 1996).

Vesicle-skin interaction studies (Figure 5) did not exhibit any major alterations except the slight fatty change in dermis revealing a decrease in resistance of skin as a barrier by fluidization of lipids, by ethanol. Stability studies performed at $4^{\circ} \mathrm{C} \pm 1^{\circ} \mathrm{C}$ for 120 days showed good storage stability (Table 4) (Dubey et al., 2007).

\section{CONCLUSION}

Soft malleable vesicles consisting of phospholipids and higher concentration of ethanol exhibited synergistic effect of phospholipids and ethanol on permeation proving elastic liposomes (ethosomes) are better carriers for Alfuzosin hydrochloride transdermal delivery. 


\section{REFERENCES}

Aqil M, Ahad A, Sultana Y, Ali A. (2007) Status of terpenes as skin penetration enhancers. Drug Discovery Today. 12, 1601-1607. [DOI]

Dayan N, Touitou E. (2000) Carriers for skin delivery of trihexyphenidyl HCL: ethosomes vs. liposomes. Biomaterials. 21, 1879-1885. [DOI]

Dubey V, Mishra D and Jain NK. (2007) Melatonin loaded ethanolic liposomes: Physicochemical characterisation and enhanced transdermal delivery. Eur. J. Pharm Biopharm. 67, 398-405. [DOI]

Dubey V, Mishra D, Nahar M, Jain V and Jain NK. (2010) Enhanced transdermal delivery of an anti-HIV agent via ethanolic liposomes. Nanomedicine: Nanotechnology, Biology, and Medicine. 6, 590-596. [DOI]

Honeywell-Nguyen PL, Bouwstra JA. (2005) Vesicles as a tool for transdermal and dermal delivery. Drug Discovery Today. 2, 67-74. [DOI]

Jain S, Tiwary AK, Sapra B, Jain NK. (2007) Formulation and Evaluation of ethosomes for transdermal delivery of Lamivudine. AAPS PharmSciTech. 8, 4, E1-E8. [DOI]

Kim KD, Choi DW, Choa YH, Kim HT. (2007) Optimisation of parameters for the synthesis of zinc oxide nanoparticles by taguchi robust design method. Colloid Surface A. 311, 170173. [DOI]

Komatsu H, Okada S. (1996) Ethanol enhanced permeation of phosphatidyl choline/phosphatidylethanolamine mixed liposomal membranes due to ethanol induced lateral phase separation. Biochim Biophys Acta. 1283, 73-79. [DOI]
Martindale. The extra pharmacopeia. $13^{\text {th }}$ Edition, [online] London, The Pharmaceutical Press. [DOI].

Panchagnula P, Salve PR, Thomas NS, Jain AK, Ramarao P. (2001) Transdermal delivery of naloxone: effect of water, propylene glycol, ethanol and their binary combinations on permeation through rat skin. Int. J. Pharm. 219, 95-105. [DOI]

Patel DB and Patel NJ. (2009) Development and validation of reverse phase high performance liquid chromatography and high performance thin layer chromatography methods for estimation of Alfuzosin hydrochloride in bulk and in pharmaceutical formulations. International Journal of Chem Tech Research. 1, 4, 985-990.

Prasanthi D and Lakshmi PK. (2012) Vesicles-mechanism of transdermal permeation: a review. Asian Journal of Pharmaceutical and clinical research. 5, 1, 18-25.

Prasanthi D, Khatry S, Shastri N, Sadanandam M.(2010) Formulation and evaluation of transdermal drug delivery systems of Alfuzosin Hydrochloride. Indian Drugs. 47, 2, 25-33.

Touitou E, Dayan N, Bergelson L, Godin B, Eliaz M. (2000) Ethosomes-novel vesicular carriers for enhanced delivery: characterization and skin penetration properties. J Control Rel. 65, 403-418. [DOI]

Verma P, Pathak K. (2012) Nanosized ethanolic vesicles loaded with econazole nitrate for the treatment of deep fungal infections through topical gel formulation: Nanomedicine: Nanotechnology, Biology, and Medicine. 8, 4, 489-496. [DOI] 